\title{
EDITORIAL
}

\section{DESARROLLO DE ENFERMERIA COMO DISCIPLINA}

\author{
Junio de 1906 se funda la primera Escuela de Enfermeras de Chile, anexa al Hospital \\ San Vicente, siendo la primera Escuela de Enfermeras de Sudamérica, institucionali- \\ zándose y profesionalizándose hace ya 94 años la formación del profesional Enferme- \\ ra/o en nuestro país.
}

A partir de ese momento, los desafíos que ha enfrentado la profesión y la disciplina no son menores, pero a través de sus organizaciones, como el Colegio de Enfermeras de Chile y la Asociación de Educación en Enfermería, es posible aseverar que Enfermería se ha consolidado para cumplir con eficacia su función social. Sin embargo, en las dos última décadas es donde ha demostrado un importante avance como disciplina, gracias a la formación de postgrado y la creación más sostenida de nuevo conocimiento, procesos donde las universidades han jugado un rol importante aportando con creatividad y perseverancia en esa dirección.

El Centro Internacional de Investigación en Enfermería (CIENF), la Clasificación Internacional para la Práctica de Enfermería (CIPE), la Comisión Internacional del Control del Abuso de Drogas (CICAD) y el Proyecto de Doctorado en Enfermería de la Universidad de Concepción son sin duda, entre otros, respuestas a la evidencia del desarrollo emergente de la disciplina de Enfermería en Chile y el mundo, y su reconocimiento por el aporte que Enfermería hace como disciplina a la salud individual y comunitaria.

La revista Ciencia y Enfermería no está ajena a esta nueva etapa en el desarrollo de la Enfermería como ciencia emergente, razón que explica algunos cambios menores en su edición que ustedes van a develar. Sin duda, absolutamente necesarios para optar a bases de datos de reconocido prestigio, acercando así cada vez más la producción del conocimiento en la disciplina a la comunidad profesional, cuyo resultado esperado es optimizar la calidad de la atención a individuos, grupos y comunidades y retroalimentar la necesaria producción de marcos conceptuales que sustenten nuestro quehacer.

\footnotetext{
DRA. JASNA STIEPOVICH BERTONI

PRESIDENTA CONSEJO DiRECTIVO REVISTA Ciencia y ENFERMERÍA
} 